The 4TU.Centre for Research Data began as a collaboration between three of the Dutch Technical Universities (Delft, Eindhoven and Twente) that form the wider 4TU Federation (which also includes Wageningen University). Since its inception in 2008, the Centre has used a variety of methods to convince and incentivise researchers to deposit their data. So far, over 7,000 datasets have been deposited, published with Digital Object Identifiers (DOIs) and are openly available for reuse.

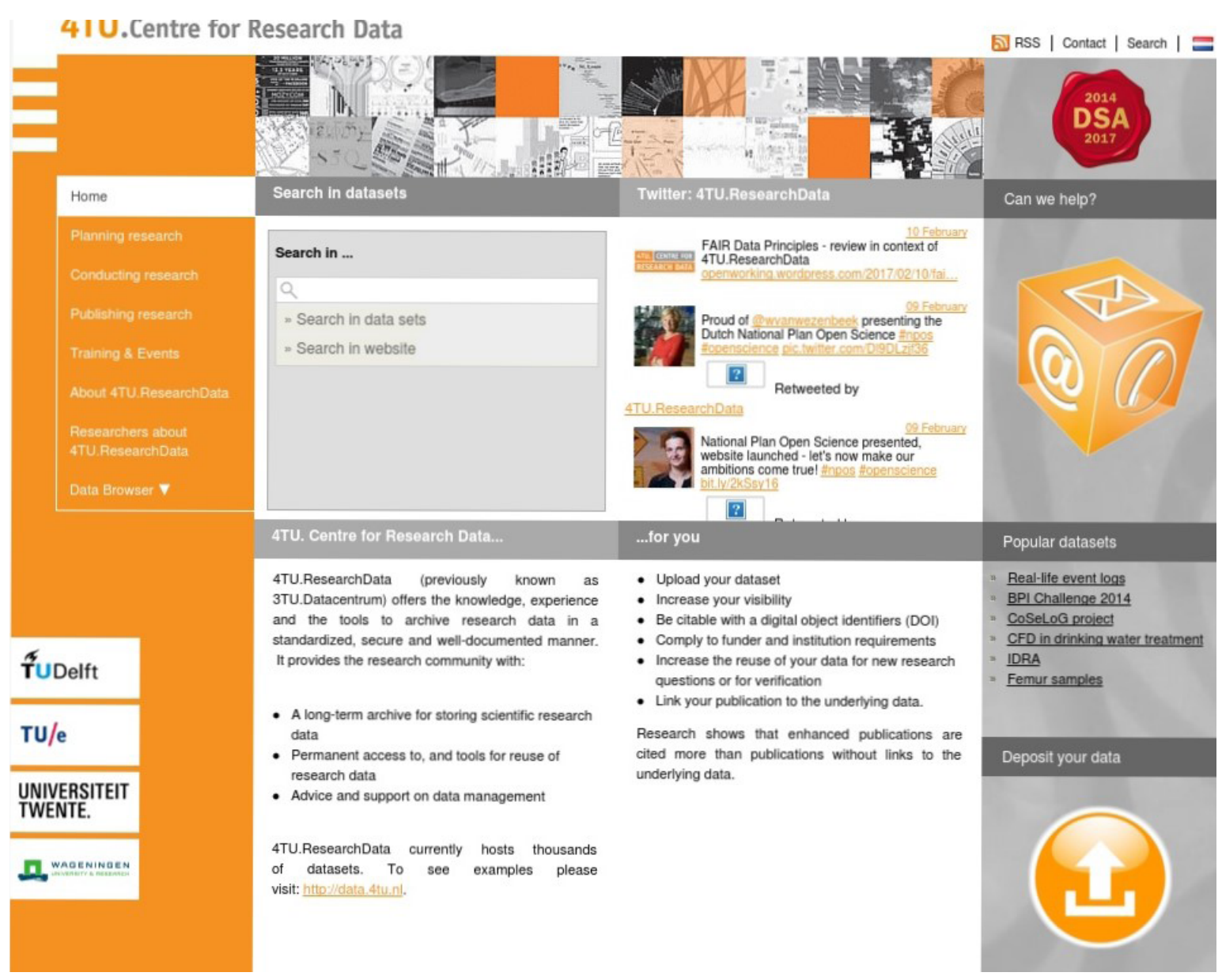

Figure 8.1 - 4TU.Centre For Research Data Homepage [http://researchdata.4tu.nl/en/homen

\subsection{METHODOLOGY}

Various methods have been used. Some have been implemented by all three of the founding universities working together, while others have been taken up by individual universities.

Case Studies on Depositing with 4TU.Research Data are available at http://researchdata.4tu.nl/en/ researchers-about-4turesearchdata/

Published case studies have demonstrated different reasons researchers have for depositing data, drawn from scientists across the 3 universities. They have been short essays (illustrated with photographs), designed for publication on the Centre's website, but also re-used in presentations and other publicity material. One case study featured the validity of the results from his team's ground-breaking experiment, that countered claims made by Einstein. Meanwhile Herman Russchenberg wanted his weather conditions data (which recorded rainfall and other meteorological events) to be used by other researchers on a global basis - this reason provided the basis of the case study.

\section{i Roadshows}

A series of lunchtime lectures has been organised for researchers within TU Delft. They have been organised and hosted at a departmental level so that staff from 4TU.ResearchData can tailor their presentation according to different disciplinary requirements. The roadshows have been developed in combination with other staff from the library. This has allowed the roadshows to present information on a variety of issues (Open Access, Current research information system implementation etc.). This has meant that more researchers have attended, so they can find out about whichever issue is pertinent to them.

\section{ii Financial Incentives}

4TU.ResearchData are currently planning to release two sets of funding to incentivise researchers from the three technical universities to deposit data. The first is a 'Data Rescue' fund. This will provide funds to researchers to allow them to prepare data so that it is suitable for depositing in the 4TU archive. Data preparation can mean giving the research team time to anonymise data, add documentation, or convert the data into formats suitable for publication.

The second is a 'Data Publication' fund. This will give researchers the time and money to write data reviews of their data for a suitable Data Journal (e.g. the Geoscience Data Journal) and provide them with suitable Article Processing Charges, if required. The data will then also be published in the 4TU archive.

\section{iii Working with ICT and Projects}

Within TU Delft, working with other partners in the university also helps spread our message. Colleagues in the ICT department often provide advice to researchers on data storage and data processing during projects. We therefore organise regular meet ups with the relevant ICT staff to inform each other about our work. This helps ensure that staff from the ICT department are also capable of passing information to researchers on why they should deposit data. 
Similarly, we also work with the research funding department of TU Delft. Given the requirements for good data management from the EU in Horizon 2020 and also from the Dutch funding agency NWO, the research funding team began to understand the importance of good data management in successful project proposals. This, in turn, has increased the likelihood of more data deposits when such projects begin to produce data.

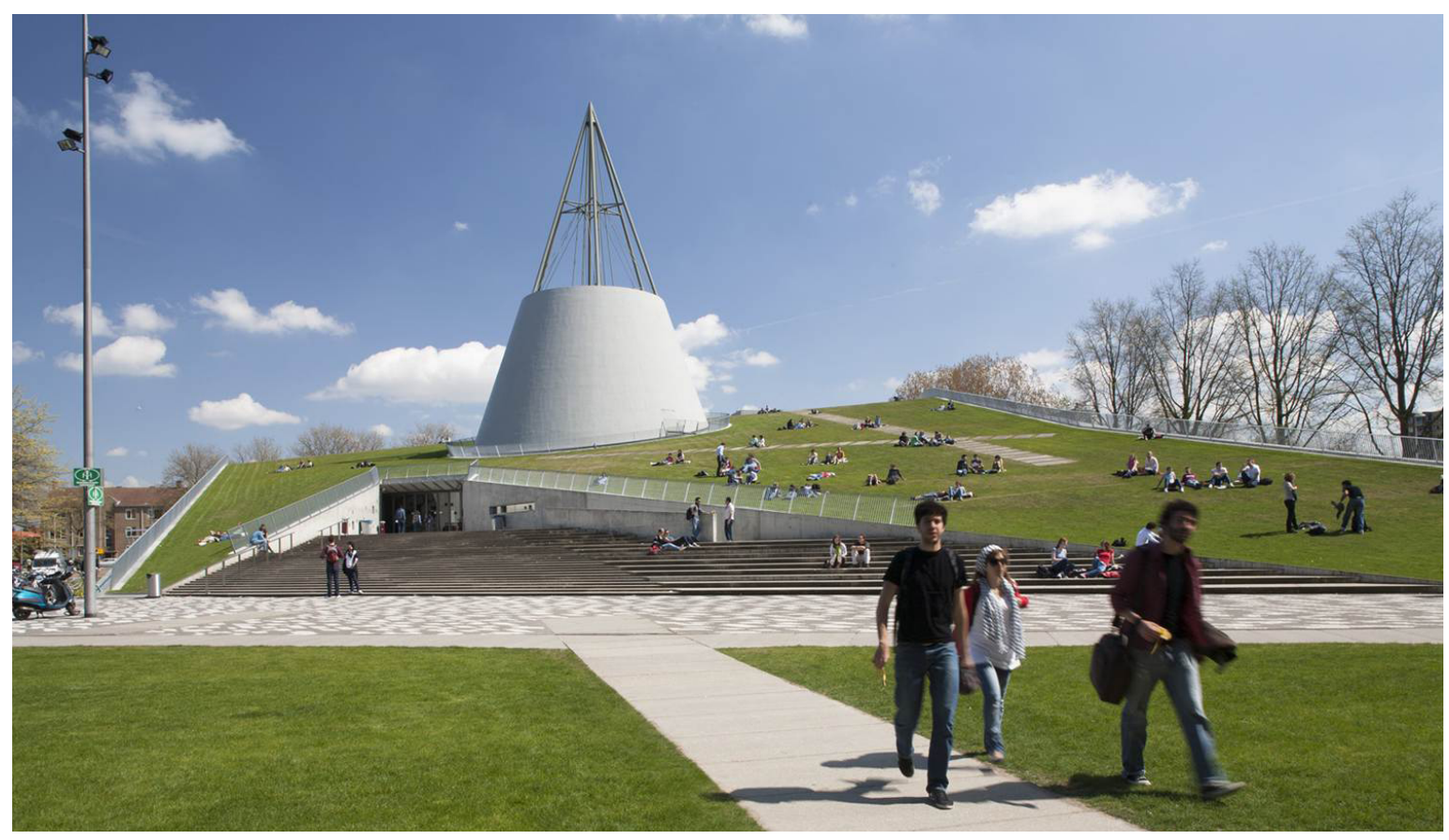

Figure 8.2 TU Delft Library, CC-BY-SA, M8Scho

\subsection{BUILDING INSTITUTION-WIDE DATA STEWARDSHIP}

While the methods referred to above have been useful, it is still a rather piecemeal approach. Given the importance of good data management to the entire scientific research lifecycle, a holistic approach was required.

Therefore the next step has been to get the entire institution to consider good data management. In 2015 , TU Delft began its Data Stewardship project, with the goal of introducing policies and best practices for data management in each of the university's eight faculties. This is being achieved with the support of the senior management of the university, who have introduced a broader Open Science programme, with the goal of promoting all types of openness in scholarly communication (e.g. open education, open access).

Being able to work with key stakeholders and persuade them why research data is important is essential. Faculty secretaries, who are the senior administrators within each of the faculties, have an important role to play here, at least in the context of TU Delft. They can help shape policy at a faculty level, but can also gauge and weigh the different responses to data management amongst a faculty's staff. This extra knowledge proves valuable in creating data management policies that can work in tandem with the researcher.

Continuing to find allies in the other support services is also important. For example, connecting with the Graduation School, which provides generic training for students, has allowed the Data Stewardship project to see how it can embed training on effective data management. This is a much longer scale piece of work to implement. It requires numerous stages, and will take a few years to complete. The four identified stages are:

\section{a) initial fact finding within faculties}

This has involved interviewing researchers and senior administrators on their attitudes and current behaviour in terms of managing their research data. Particular attention has been paid to the varying practices and methodologies within different disciplines, and the impact these have on data management.

\section{b) development of a draft policy}

Based on the above, a draft policy was written stating potential roles and responsibilities for stakeholders within the university. It also offered faculties specific options on how they would deal with the following three areas: training for $\mathrm{PhD}$ students, data management plans and training for researchers.

\section{c) ongoing conversations about implementing such a policy within separate faculties}

The draft policy is then used to continue discussion on the implementation of good data management, with a focus on the staff and infrastructure required at each stage of the research lifecycle.

\section{d) implementation of processes and policies}

It is envisioned that there will be funds made available to allow the faculties to put into practice the demands made in the university-wide policy, for example with regard to PhD training or the creation of individual Data Management Plans for each project. Most importantly, it is hoped that Data Stewards can be embedded in each faculty to provide tailor made help for the different disciplines within the university.

At time of writing the second phase of activity has just been completed.

\subsection{CONCLUSION}

The 4TU.Centre for Research Data has been in existence for nearly ten years. It has therefore had ample opportunity to explore various methods for incentivising researchers to share their data.

While they have limited reach, the early steps taken (roadshows, published case studies) are essential to get initial contact with stakeholders in the university. The most likely way one has of convincing researchers to deposit their data is if their disciplinary peers are already doing this. Therefore the local case studies are important, offering personal testimony. Roadshows are also important as the face to face contact helps build trust, and gets Library staff out of the Library and into the faculties and departments.

However, to advance all this to the next level, a wider institutional approach is required. TU Delft's Data Stewardship project identifies and works with key influencers throughout the university. Engaging the necessary stakeholders and implementing policies - as opposed to engaging individual researchers on a one to one basis - is essential for any university wishing to see Data Stewardship work at scale.

This project has received funding from the Europ 